n.tronos

glyndîn

Glyndŵr University

Glyndŵr University Research Online

Computing

Computer Science

$10-1-2002$

\title{
Three-dimensional space-borne synthetic aperture radar (SAR) imaging with multiple pass processing
}

Zhishun She

Glyndwr University, z.she@glyndwr.ac.uk

D A. Gray

RE. Bogner

J Homer

ID. Longstaff

Follow this and additional works at: http://epubs.glyndwr.ac.uk/cair

Part of the Computer Engineering Commons

\section{Recommended Citation}

She, Z., Gray, D. A., Bogner, R. E., Homer, J., \& Longstaff, I. D. (2002)‘Three-dimensional space-borne synthetic aperture radar (SAR) imaging with multiple pass processing. International Journal Remote Sensing, 23(20), 4357-4382

This Article is brought to you for free and open access by the Computer Science at Glyndŵr University Research Online. It has been accepted for inclusion in Computing by an authorized administrator of Glyndŵr University Research Online. For more information, please contact

d.jepson@glyndwr.ac.uk. 


\title{
Three-dimensional space-borne synthetic aperture radar (SAR) imaging with multiple pass processing
}

\begin{abstract}
Three-dimensional (3D) synthetic aperture radar (SAR) imaging via multiple-pass processing is an extension of interferometric SAR imaging. It exploits more than two flight passes to achieve a desired resolution in elevation. In this paper, a novel approach is developed to reconstruct a 3D space-borne SAR image with multiple-pass processing. It involves image registration, phase correction and elevational imaging. An image model matching is developed for multiple image registration, an eigenvector method is proposed for the phase correction and the elevational imaging is conducted using a Fourier transform or a super-resolution method for enhancement of elevational resolution. 3D SAR images are obtained by processing simulated data and real data from the first European Remote Sensing satellite (ERS-1) with the proposed approaches.
\end{abstract}

\section{Keywords}

3D SAR, multiple pass processing, ERS-1, super-resolution

\section{Disciplines}

Computer Engineering

\section{Comments}

Reproduced by permission of the publisher. (C) 2002 Taylor and Francis. This is an electronic version of an article published in She, Z., Gray, D. A., Bogner, R. E., Homer, J., \& Longstaff, I. D. (2002)‘Three-dimensional space-borne synthetic aperture radar (SAR) imaging with multiple pass processing'. International Journal Remote Sensing, 23(20), 4357-4382. The final version of the article as published in the print edition of the International Journal of Remote Sensing by Taylor and Francis is available online at

http://www.informaworld.com 
INT. J. REMOTE SENSING, 2002, VOL. 23, NO. 20, 4357-4382

\title{
Three-dimensional space-borne synthetic aperture radar (SAR) imaging with multiple pass processing
}

\author{
Z. SHE* \\ School of Informatics, University of Wales, Bangor, Gwynedd, LL57 1UT, \\ Wales, UK
}

D. A. GRAY, R. E. BOGNER

Cooperative Research Centre for Sensor Signal and Information Processing (CSSIP) and Department of Electrical and Electronic Engineering, The University of Adelaide, Adelaide, SA 5005, Australia

\section{J. HOMER and I. D. LONGSTAFF}

CSSIP and Department of Computer Science and Electrical Engineering, The University of Queensland, Brisbane, QLD 4072, Australia

(Received 28 June 2000; in final form 2 April 2002)

\begin{abstract}
Three-dimensional (3D) synthetic aperture radar (SAR) imaging via multiple-pass processing is an extension of interferometric SAR imaging. It exploits more than two flight passes to achieve a desired resolution in elevation. In this paper, a novel approach is developed to reconstruct a 3D space-borne SAR image with multiple-pass processing. It involves image registration, phase correction and elevational imaging. An image model matching is developed for multiple image registration, an eigenvector method is proposed for the phase correction and the elevational imaging is conducted using a Fourier transform or a super-resolution method for enhancement of elevational resolution. 3D SAR images are obtained by processing simulated data and real data from the first European Remote Sensing satellite (ERS-1) with the proposed approaches.
\end{abstract}

\section{Introduction}

Synthetic aperture radar (SAR) is a high-resolution coherent radar. It is usually carried on an aircraft or a satellite to map terrain in range and azimuthal directions which results in a two-dimensional (2D) high-resolution image (Wehner 1995). A wide-band signal is transmitted to achieve high range resolution and a large antenna aperture synthesized by motion of the radar provides high azimuthal resolution. Since its origins in the 1950s, SAR has been extensively developed and its all-weather high-resolution imaging capability has been used for civilian and surveillance applications.

Interferometric SAR (InSAR) is a technique to extract elevational information about the mapped terrain from two complex SAR images (Graham 1974, Zebker

\footnotetext{
*Corresponding author; e-mail: zshe@sees.bangor.ac.uk
} 
and Goldstein 1986, Gabriel and Goldstein 1988, Goldstein et al. 1988). The InSAR processing first registers two complex SAR images and secondly forms an interferogram by multiplying one registered image with the conjugate of the other. It then unwraps the principal phase value of the interferogram, and finally reconstructs a digital elevation model (DEM) of terrain. However, the 2D phase unwrapping is restricted by SAR image decorrelation (Zebker and Villasenor 1992), the generation of DEM is dependent on precise baseline estimation (Rufino et al. 1998) and the reflectivity of the terrain is unknown.

Multiple-pass SAR (MPSAR) is a natural extension of typical InSAR. It makes use of more than two SAR imaging flight passes to synthesize an aperture in elevation, resulting in improved elevational resolving power (Pasquali et al. 1995, Homer et al. 1996, 1997, She et al. 1999, Reigber et al. 2000). The elevational imaging, combined with single-pass SAR imagery in the range and azimuthal directions, constructs a three-dimensional (3D) SAR image. This image provides information about both the elevational variation and the reflectivity of terrain, which is important for terrain mapping, analysis and classification.

The principle of 3D SAR imaging originated from 3D holographic processing (Wolf 1969). The basic idea of 3D SAR imaging was described in the literature (Walker 1980, Chan and Farhat 1981, Ausherman et al. 1984, Farhat 1984). Since then some investigations have been conducted. However, most of them were carried out with simulated data ( $\mathrm{Li}$ et al. 1997) or under ideal experimental conditions (Fortuny and Sieber 1999). Recently 3D SAR imaging has been reported for airborne SAR processing (Reigber et al. 2000). In the study, the position of each flight path needed to be known or to be measured precisely with inertial navigation systems (INS) and global position systems (GPS). Nevertheless in practical applications the positions of flight paths are unknown and the size of flight path array is limited. Sometimes the accuracy of measurement cannot meet the requirement (a fraction of radar wavelength) for 3D SAR imaging and the enlarged array aperture is requested for high elevational resolution. Meanwhile sensor array processing techniques have been extensively developed in the past two decades (Krim and Viberg 1996). The multiple flight paths form a synthesized array in the elevational direction and this synthesized array can be solved from the perspective of array processing. The contributions of this paper are to investigate multiple SAR image registration, apply the array calibration technique (Rockah and Schuitheiss 1987) to estimate the shape of unknown flight path array and extend the physical limitation of this array by super-resolution processing (Kay and Marple 1981) in the case of space-borne SAR processing.

\section{Mathematical model}

The geometry of 3D SAR imaging is shown in figure 1 where $x, y$ and $z$ represent range, azimuth and elevation, respectively, and $P_{i} i=1, \ldots, N$ denotes $i$ th flight path. They are assumed to be parallel and equi-spaced in elevation. Consider a terrain patch whose centre is taken as the origin. The patch is supposed to be a rigid body with a finite number of point scatterers spread on its surface, each having its own elevation. The slant ranges from the closest point B of approach for each flight path to the patch centre $o$ and scene point A with coordinate $(x, y, z)$ are denoted by $R_{0}$ and $R$, respectively. 


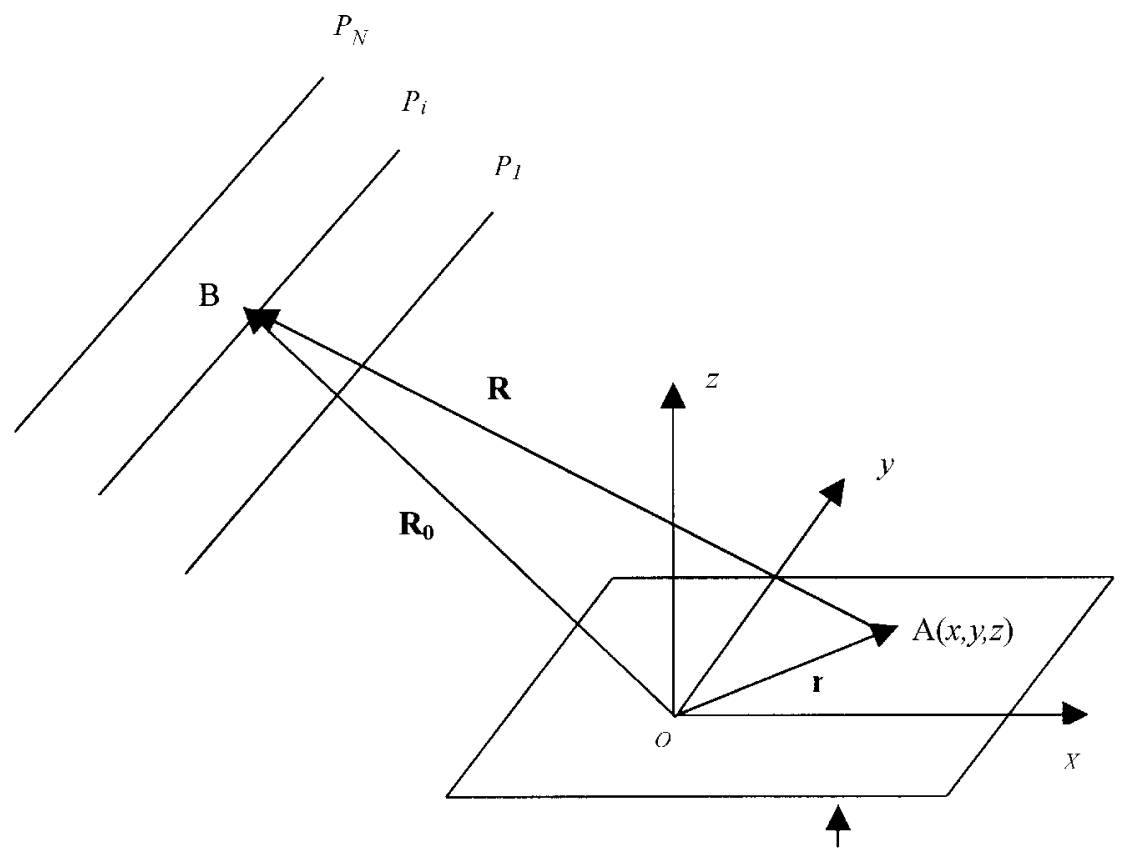

Terrain Patch

Figure 1. 3D SAR imaging geometry.

For a transmitted signal, $u(t)$, the received signal, $s_{r}\left(t, R_{0}\right)$, from point scatterer A is (Skolink 1990)

$$
s_{r}\left(t, R_{0}\right)=\sigma(x, y, z) u\left(t-\frac{2 R(x, y, z)}{c}\right)
$$

where $\sigma(x, y, z)$ is the reflectivity of $\mathrm{A}$, which is assumed to be independent of frequency and aspect angle, and $c$ the propagation speed of light. The total of the received signals from all scatterers of the distributed object is

$$
s_{r}\left(t, R_{0}\right)=\int_{V} \sigma(x, y, z) u\left(t-\frac{2 R(x, y, z)}{c}\right) \mathrm{d} x \mathrm{~d} y \mathrm{~d} z
$$

where $\sigma(x, y, z)$ is the reflectivity density, which includes propagation effects and various system gains for convenience. The integration is carried out over a terrain volume $V$. If we take the Fourier transform of equation (2) with respect to $t$, we obtain

$$
S_{r}\left(f, R_{0}\right)=U(f) \int_{V} \sigma(x, y, z) \exp [-j 4 \pi f R(x, y, z) / c] \mathrm{d} x \mathrm{~d} y \mathrm{~d} z
$$

where $U(f)$ is the complex Fourier spectrum of the transmitted signal. The output of a filter $S_{r e f}\left(f, R_{0}\right)=U^{*}(f) \exp \left(j 4 \pi f R_{0} / c\right)$ matched to a reflection of the transmitted wavefront from an ideal scatterer at the origin $o$ for focusing is given by

$$
S_{o}\left(f, R_{0}\right)=|U(f)|^{2} \int_{V} \sigma(x, y, z) \exp \left\{-j 4 \pi f\left[R(x, y, z)-R_{0}\right] / c\right\} \mathrm{d} x \mathrm{~d} y \mathrm{~d} z
$$


If the range of the terrain is large compared with the size of the terrain patch, that is, $R \gg r$, we have an approximation

$$
R(x, y, z) \cong R_{0}-\frac{\mathbf{r}(x, y, z) \cdot \mathbf{R}_{0}}{R_{0}}
$$

where $\mathbf{r}(x, y, z)$ and $\mathbf{R}_{0}$ are the corresponding range vectors as shown in figure 1. Define a vector $\boldsymbol{q}$ of the reference point as

$$
\boldsymbol{q}=\frac{2 f \mathbf{R}_{0}}{c R_{0}}
$$

For a specific frequency, $\boldsymbol{q}$ lies on the surface of a sphere as $\mathbf{R}_{0}$ is varied. For various frequencies, $\boldsymbol{q}$ is mapped into a vector in a $3 \mathrm{D}$ data space $\left(q_{x}, q_{y}, q_{z}\right)$. Thus we have

$$
S_{o}(\boldsymbol{q})=|U(f)|^{2} \int_{V} \sigma(x, y, z) \exp \{j 2 \pi \boldsymbol{q} \cdot \mathbf{r}(x, y, z)\} \mathrm{d} x \mathrm{~d} y \mathrm{~d} z
$$

This implies that except for the factor $|U(f)|^{2}$ the output of focusing is a finite inverse 3D Fourier transform of the terrain reflectivity function $\sigma(x, y, z)$. This model is consistent with the results in Ausherman et al. (1984) and Marechal (1995).

The support region of $\boldsymbol{q}$ can be shown in the data domain (Walker 1980). Each pulse is corresponding to a radial line as shown in figure 2(a). A single flight path results in a shaded section region as shown in figure $2(a)$. Multiple-pass processing produces a volume in a 3D data space $V_{f}$ which is composed of slices of $2 \mathrm{D}$ data recorded for individual flight paths as shown in figure 2(b). Letting $\boldsymbol{q}=\left(q_{x}, q_{y}, q_{z}\right)$ and $\mathbf{r}=(x, y, z)$ then $\sigma(x, y, z)$ can be estimated by the 3D Fourier transform of $S_{o}\left(q_{x}, q_{y}, q_{z}\right) /|U(f)|^{2}$ over a limited support region $V_{f}$, that is,

$$
\sigma(x, y, z)=\int_{q_{x}} \int_{q_{y}} \int_{q_{z}} \frac{S_{o}\left(q_{x}, q_{y}, q_{z}\right)}{|U(f)|^{2}} \exp \left\{-j 2 \pi\left(x q_{x}+y q_{y}+z q_{z}\right)\right\} \mathrm{d} q_{x} \mathrm{~d} q_{y} \mathrm{~d} q_{z}
$$

as shown in figure $2(c)$.

Equation (8) indicates that the 3D reflectivity function $\sigma(x, y, z)$ can be estimated by three one-dimensional (1D) Fourier transforms which are realized by wideband pulse compression in $x$ direction, azimuthal processing in $y$ direction and elevational

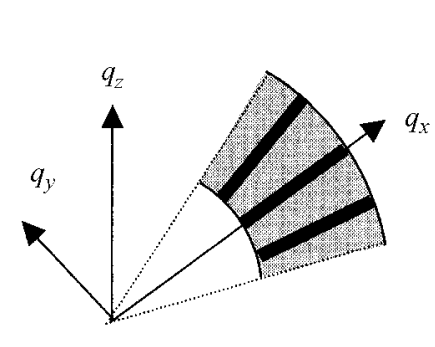

(a) single flight path data

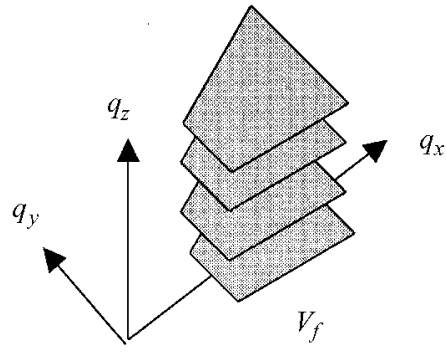

(b) multiple flight path data

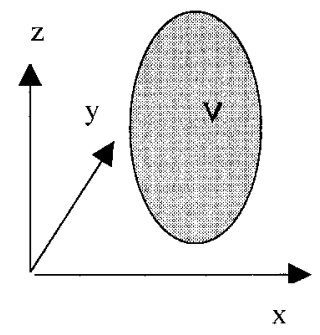

(c) image of object

Figure 2. 3D data and image space. 
processing in $z$ direction. If the complex image of each flight path is available, equation (8) can be changed into

$$
\sigma(x, y, z)=\int_{q_{z}} G^{\prime}\left(x, y, q_{z}\right) \exp \left\{-j 2 \pi z q_{z}\right\} \mathrm{d} q_{z}
$$

where

$$
G^{\prime}\left(x, y, q_{z}\right)=\int_{q_{x}} \int_{q_{y}} S_{o}\left(q_{x}, q_{y}, q_{z}\right) /|U(f)|^{2} \exp \left\{-j 2 \pi\left(x q_{x}+y q_{y}\right)\right\} \mathrm{d} q_{y} \mathrm{~d} q_{x}
$$

represents a SAR complex image of a single flight pass after focusing. Equation (9) shows that with multiple-pass processing of SAR, $\sigma(x, y, z)$, the $3 \mathrm{D}$ image, can be estimated by doing a 1D Fourier transform in each pixel cell of the multiple SAR complex image set. In practice, we only have discrete flight passes. Thus we have

$$
\sigma(x, y, z)=\sum_{i=1}^{N} G_{i}^{\prime}(x, y) \exp \left\{-j 2 \pi z q_{i}\right\}
$$

where $G_{i}^{\prime}(x, y)$ denotes the complex SAR image of $i$ th flight path after phase correction and $q_{i}$ is determined by the position of $i$ th flight path. In general, equation (10) represents an $N$-point discrete Fourier transform (DFT). If the flight paths are equi-spaced in elevation, equation (10) can be implemented with an $N$-point fast Fourier transform (FFT).

Based on theory of spectral analysis the spacing of adjacent flight path $d$ determines the maximal elevational distance $z_{\max }$ that can be unambiguously estimated via the relationship

$$
z_{\max }=\frac{\lambda R_{0}}{2 d}
$$

where $\lambda$ denotes radar wavelength. The elevational resolution $r_{z}$ depends on the aperture length $D=(N-1) d$ in the elevational direction by

$$
r_{z}=\frac{\lambda R_{0}}{2 D}
$$

For a given number of flight paths, a small baseline $d$ will reduce the ambiguity problem. However, this will also reduce the elevational resolution. There is a compromise between elevational ambiguity and resolution. The baseline distribution needs to be designed for specific requirement.

\section{3D SAR imaging approach}

Each flight pass performs conventional SAR processing including complex I, Q data collection, range compression, quadratic phase correction and azimuthal compression, which results in multiple complex SAR images. However rather than forming absolute value of the complex image at the last step, 3D SAR imaging uses each SAR image in its complex form. Clearly the $N$ complex SAR images $G_{i}(x, y)$ have different phases for different values of $i$, which contain the information about the reflectivity distribution along the elevational direction. The main function of $3 \mathrm{D}$ SAR imaging is to extract the reflectivity distribution in the elevation from the $N$ 
complex SAR images. In its simplest form, the elevational imaging involves a straightforward Fourier transform of $N$ points along the $i$-axis of $G_{i}^{\prime}(x, y)$ as shown in equation (10).

In the analysis of $\S 2$, the motion of the SAR platform is assumed to be known so that focusing is conducted precisely. However, in practical applications, the flight path is unknown or not known precisely, causing individual complex SAR images to be misaligned in range and azimuth directions. This uncertainty of the flight path induces an unknown phase factor, and thus each complex SAR image $G_{i}(x, y)$ needs to be calibrated or corrected in phase prior to elevational imaging, namely,

$$
\sigma(x, y, z)=\sum_{i=1}^{N} G_{i}(x, y) \exp \left\{j \phi_{i}\right\} \exp \left\{-j 2 \pi z q_{i}\right\}
$$

where $\phi_{i}$ is the phase for correction caused by the uncertainty of each flight path.

Based on the above analysis, a novel approach to 3D SAR imaging via multiplepass processing is developed. The block diagram as shown in figure 3 includes the following steps:

1. It carries out conventional SAR imaging for each flight path.

2. It registers multiple complex SAR images, which aligns the returns resulted from the common terrain in the multiple images into the same image pixel cell.

3. It corrects the registered SAR images in phase which multiplies each image by a phase factor to make the phases of the multiple complex images cohere at the reference point of the terrain.

4. It re-samples the unequal spacing flight paths and then performs an $N$-point FFT across the equi-spaced flight paths. This operation is the core of 3D SAR imaging where FFT acts as an elevational filter with the output of each FFT bin giving the reflectivity whose elevation is within a particular range.

Essentially, three orthogonal axes (range, azimuth and elevation) out of the 3D SAR image field may be visualized by three $2 \mathrm{D}$ images in the range-azimuth (top view), range-elevation (front view) and azimuth-elevation (side view) planes. The elevation resolution can be illustrated in the range-elevation and azimuthelevation images.

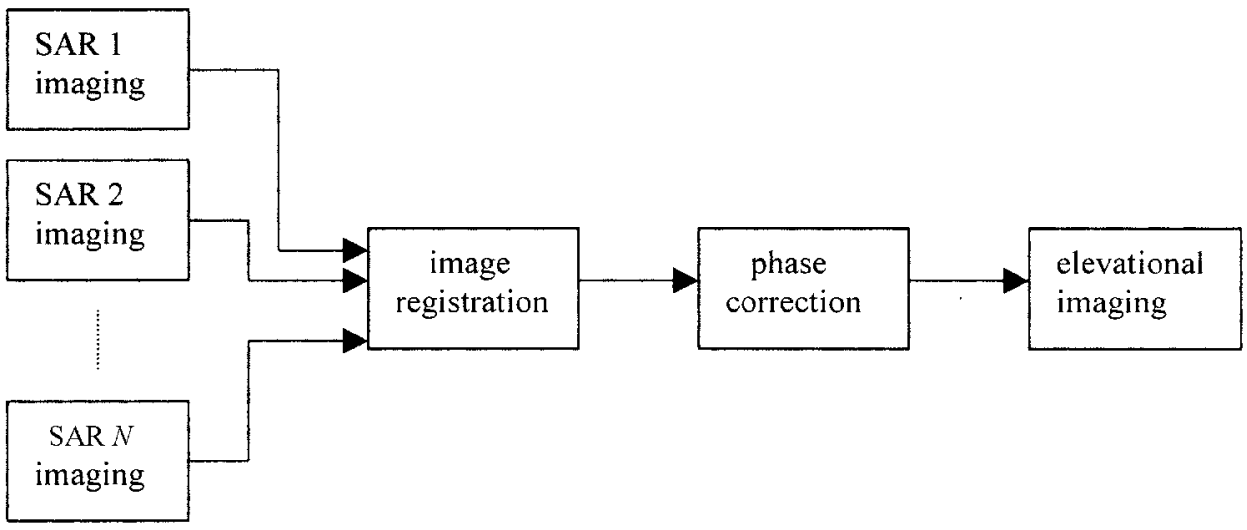

Figure 3. Block diagram of 3D SAR imaging. 
In $\S 2$, a reference function is applied to a patch of image rather than each pixel in order to reduce computational complexity of focusing. However, the size of patch is limited by the phase error tolerance of SAR system and needs to be determined. When the image registration is accomplished, the multiple SAR images are realigned in range and azimuth directions so that the signal returns from multiple flight paths resulting from the same terrain will be located in the same image pixel cell. After phase correction, the focused beam in elevation is formed and it points to the reference point of the terrain patch. Using the DFT applies a linear phase across the synthesized elevational aperture to steer beam away from the reference point. This introduces a phase error due to the linear approximation for $R$ in equation (5). The appropriate size of terrain patch can be determined by limiting the phase error. According to figure 1, we have

$$
R=\left(R_{0}^{2}+r^{2}-2 \mathbf{R}_{0} \cdot \mathbf{r}\right)^{1 / 2}
$$

The residual error $\Delta R$ in equation (5) is less than $r^{2} /\left(2 R_{0}\right)$, that is,

$$
\Delta R<r^{2} /\left(2 R_{0}\right)
$$

If we restrain $\Delta R$ to be less than $\lambda / 8$, we have

$$
R_{0}>4 r^{2} / \lambda
$$

Thus the size of terrain patch $r_{\max }$ can be determined by

$$
r_{\max }=\left(R_{0} \lambda\right)^{1 / 2} / 2
$$

As an example of ERS-1 system, we have a satellite altitude of $785 \mathrm{~km}$, an incidence angle of $23^{\circ}$, and a wavelength of $5.67 \mathrm{~cm}$, thus $r_{\max }=110 \mathrm{~m}$.

Although the developed proposed approach is suitable for a small area, it can be extended to 3D SAR imaging of a large area by use of subpatch processing. First a large area which breaks the limitation of equation (17) is divided into many small patches that satisfy equation (17). Then the 3D SAR imaging is conducted by the approach described above for each divided patch. Finally the $3 \mathrm{D}$ SAR image of the large area can be generated by mosaicking the reconstructed $3 \mathrm{D}$ images of small patches.

\section{Multiple image registration}

The geometrical transformations between SAR images of repeated passes are identified as local translations in range and azimuth ( $\mathrm{Li}$ and Goldstein 1990, Jakowatz et al. 1996). In order to correct the local translational distortion, image registration for SAR images is usually accomplished in four steps. First, the SAR image is divided into many subimages. Secondly, measurements are made of the local misregistrations from one image to another in all subimages. Thirdly, the misregistrations are then used to calculate a warping function, which maps a location in one image to the corresponding location in the other. Finally, the first image is resampled so that it overlays the second precisely.

The crux of the registration process is to determine the spatial misregistrations between one subimage and another. The existing methods to estimate misregistration are applicable to image pairs. Multiple images are registered by a process of repeating the operation until all the images are registered. However, the registration result is dependent on the order of the images and it suffers from image drift error resulting from the error accumulation of correlation and image variation error caused by 
decorrelation of SAR images. In order to reduce these errors, the correlation of all the images in one step rather than the correlation of image pairs should be used. In this section, a minimal distance approach is firstly proposed, and then an image model matching approach is developed to improve computational efficiency.

\subsection{Minimal distance approach}

Define a distance measure $\mathrm{D}\left[G_{i}(x, y), G_{l}(x, y)\right]$ between two images $G_{i}(x, y)$ and $G_{l}(x, y)$ satisfying

$$
\begin{aligned}
& \mathrm{D}\left[G_{i}(x, y), G_{l}(x, y)\right]>0 \forall G_{i}(x, y) \neq G_{l}(x, y) \\
& \mathrm{D}\left[G_{i}(x, y), G_{i}(x, y)\right]=0 \\
& \mathrm{D}\left[G_{i}(x, y), G_{l}(x, y)\right]=\mathrm{D}\left[G_{l}(x, y), G_{i}(x, y)\right] \\
& \mathrm{D}\left[G_{i}(x, y), G_{l}(x, y)+G_{k}(x, y)\right]<\mathrm{D}\left[G_{i}(x, y), G_{l}(x, y)\right]+\mathrm{D}\left[G_{i}(x, y), G_{k}(x, y)\right]
\end{aligned}
$$

Let $\Delta x_{i}$ and $\Delta y_{i} i=1, \ldots, N$ be the misregistrations of the ith image $G_{i}(x, y)$ in the range and azimuthal directions, respectively. Multiple image registration depends on a cost function

$$
C_{1}\left(\Delta x_{1}, \Delta y_{1}, \ldots, \Delta x_{N}, \Delta y_{N}\right)=\sum_{i=1}^{N} \sum_{l=i^{+}}^{N} \mathrm{D}\left[G_{i}\left(x+\Delta x_{i}, y+\Delta y_{i}\right), G_{l}\left(x+\Delta x_{l}, y+\Delta y_{l}\right)\right]
$$

where $G_{i}\left(x+\Delta x_{i}, y+\Delta y_{i}\right)$ is the image of $G_{i}(x, y)$ with shifts $\Delta x_{i}$ and $\Delta y_{i}$ in range and azimuthal directions, respectively. The misregistrations of multiple images can be determined by minimizing equation (19) with respect to all the $\Delta x_{i}, \Delta y_{i}$, that is,

$$
\left(\Delta x_{1}^{0}, \Delta y_{1}^{0}, \ldots, \Delta x_{N}^{0}, \Delta y_{N}^{0}\right)=\operatorname{Argmin}\left(C_{1}\left(\Delta x_{1}, \Delta y_{1}, \ldots, \Delta x_{N}, \Delta y_{N}\right)\right)
$$

which is called the minimal distance method.

The minimal distance method considers the correlation of multiple images to be processed rather than the correlation of image pairs. It is less affected by the SAR image decorrelation than the conventional method for image pairs. Moreover, as the method calculates the correlation as a whole instead of computing the multiple correlations between two images, it avoids the problem of error accumulation in correlation estimation and the effect of image order. However, the computational load of the minimal distance method is very expensive because it requires the optimization of multiple variables. Although it is a good method for multiple image registration, it is impractical especially when the number of images is large.

\subsection{Image model matching approach}

A new algorithm for multiple image registration named image model matching is developed for practical applications. It is assumed that each image is derived from a reference image $M(x, y)$ by the addition of noise. The new cost function of multiple image registration is expressed as

$$
C_{2}\left(\Delta x_{1}, \Delta y_{1}, \ldots, \Delta x_{N}, \Delta y_{N}\right)=\sum_{i=1}^{N} \mathrm{D}\left[G_{i}\left(x+\Delta x_{i}, y+\Delta y_{i}\right), M(x, y)\right]
$$


Minimizing equation (21) is equivalent to minimizing the distance of each image to the reference image, that is,

$$
\min \left[C_{2}\left(\Delta x_{1}, \Delta y_{1}, \ldots, \Delta x_{N}, \Delta y_{N}\right)\right]=\sum_{i=1}^{N} \min \left\{\mathrm{D}\left[G_{i}\left(x+\Delta x_{i}, y+\Delta y_{i}\right), M(x, y)\right]\right\}
$$

The misregistrations can be estimated by

$$
\left(\Delta x_{1}^{0}, \Delta y_{1}^{0}, \ldots, \Delta x_{N}^{0}, \Delta y_{N}^{0}\right)=\operatorname{Argmin}\left(C_{2}\left(\Delta x_{1}, \Delta y_{1}, \ldots, \Delta x_{N}, \Delta y_{N}\right)\right)
$$

It is noted that the optimization of $2 N$ variables in equation (20) is simplified into the $N$ optimizations of two variables in equation (22). Since the dimension of optimization space is changed from $2 N$ into 2, the computational burden of the image model matching approach is greatly reduced compared with the minimal distance approach.

The image model matching method matches each image to the reference image rather than each other image so that the effects of image order and image drift are eliminated and the effect of image variation is reduced. The critical step of image model matching method is how to estimate the reference image. We first measure the $N(N-1) / 2$ distances between all image pairs to determine the misregistrations and distances. The initial reference image $M_{1}(x, y)$ is formed by summing two registered images with the minimal distance, i.e.

$$
M_{1}(x, y)=\operatorname{sum}\left[G_{k}\left(x+\Delta x_{k}, y+\Delta y_{k}\right), G_{l}\left(x+\Delta x_{l}, y+\Delta y_{l}\right)\right]
$$

where $k, l=\operatorname{Argmin}\left[\mathrm{D}\left(G_{i}\left(x+\Delta x_{l}, y+\Delta y_{i}\right), G_{m}\left(x+\Delta x_{m}, y+\Delta y_{m}\right)\right)\right] \forall i \neq m i, m=1$, $\ldots, N$. When signal-to-noise ratio (SNR) is high, the two images can be accumulated coherently after removing the interferometric phase. If SNR is low, they are added incoherently.

Then the remaining images are reordered as $G_{i}(x, y) i=1, \ldots, N-2$ and are matched to $M_{1}(x, y)$ to determine the misregistrations and distances. The reference image $M_{2}(x, y)$ is produced by summing $M_{1}(x, y)$ with one registered image which has a minimal distance with $M_{1}(x, y)$, namely,

$$
M_{2}(x, y)=\operatorname{sum}\left[M_{1}(x, y), G_{k}\left(x+\Delta x_{k}, y+\Delta y_{k}\right)\right]
$$

where $k=\operatorname{Argmin}\left[\mathrm{D}\left(G_{i}\left(x+\Delta x_{i}, y+\Delta y_{i}\right), M_{1}(x, y)\right)\right] \forall i=1, \ldots, N-2$. The above procedure is repeated $N-2$ times until the reference image $M_{N^{-}}{ }_{1}(x, y)$ is produced and images $G_{i}(x, y) i=1, \ldots, N$ have been registered.

We can further elaborate the reference image to increase the accuracy of multiple image registration by iterating the match of the reference image to each image $G_{i}(x, y)$ until the misregistrations $\Delta x_{i}, \Delta y_{i} i=1, \ldots, N$ remain constant.

\section{Phase correction}

In $\S 2$, it is assumed that the multiple flight paths are parallel and equi-spaced in elevation and thus the beam output formed by summing the registered images enhances a plane wavefront signal incident on the flight path array from the broadside direction of elevation. However, in practice, the flight paths are uncertain and deviate from their nominal position and the propagation medium of signals in ionosphere and troposphere may be inhomogeneous (Zebker et al. 1997). These defects may destroy the above capability of coherent summation. In order to correct the uncertainty of flight paths and compensate for anomalies in the propagation medium, a 
phase shift is required to be attached to each flight path to form a focused beam in the elevation. The phase correction is dependent upon precise information on flight paths, propagation medium and terrain slope (Gatelli et al. 1994). In this section, an approach to phase correction is developed based on array self-calibration which does not need the information about flight paths, propagation medium and terrain slope.

The subimage after image registration can be modelled by ( $\mathrm{Li}$ and Goldstein 1990, Gatelli et al. 1994)

$$
G_{2}(x, y)=G_{1}(x, y) \exp \left\{-j \varphi_{21}(x, y)\right\}
$$

where $G_{1}(x, y)$ and $G_{2}(x, y)$ are the subimages of the first and the second flight path, respectively, $\varphi_{21}(x, y)$ the unknown phase variation caused by different flight paths, propagation medium and terrain slope. For the complex image of the $i$ th flight path, equation (26) generalizes to

$$
G_{i}(x, y)=G_{i^{-}}{ }_{1}(x, y) \exp \left\{-j \varphi_{i, i^{-}}{ }_{1}(x, y)\right\}=G_{1}(x, y) \exp \left\{-j \phi_{i}(x, y)\right\}
$$

where

$$
\phi_{i}(x, y)=\sum_{n=2}^{i} \varphi_{n, n^{-} 1}(x, y)
$$

Defining a pixel vector $V(x, y)=\left[G_{1}(x, y), \ldots, G_{N}(x, y)\right]^{T}$ and considering the SAR system noise, equation (27) has the form for each pixel

$$
V(x, y)=G_{1}(x, y) D(x, y)+W(x, y)
$$

where $D(x, y)=\left[1, \exp \left\{-j \phi_{2}(x, y)\right\}, \ldots, \exp \left\{-j \phi_{N}(x, y)\right\}\right]^{T}$ and $W(x, y)=\left[w_{1}(x, y), \ldots\right.$, $\left.w_{N}(x, y)\right]^{T} \cdot w_{i}(x, y)$ is assumed to be independent identically distributed complex Gaussian noise components with zero mean and variance $\sigma_{w}^{2}$. The tasks of phase correction are to first estimate the complex signal vector $D(x, y)$, and then to compensate the multiple complex SAR images by conjugating with $D(x, y)$.

The covariance matrix of $V(x, y)$ is

$$
C_{V}(x, y)=\mathrm{E}\left\{V(x, y) V^{\mathrm{H}}(x, y)\right\}=\left|G_{1}(x, y)\right|^{2} D(x, y) D^{\mathrm{H}}(x, y)+\sigma_{w}^{2} I
$$

where $I$ is the identity matrix, E the ensemble average and $\mathrm{H}$ the complex conjugate transpose. It can be verified that the largest eigenvalue of $C_{v}(x, y)$ is

$$
\lambda_{1}=\left|G_{1}(x, y)\right|^{2} N+\sigma_{w}^{2}
$$

and its corresponding normalized eigenvector is

$$
u_{1}=D(x, y) / N
$$

and all the other eigenvalues are equal to $\sigma_{w}^{2}$. Equation (31) shows that the eigenvector corresponding to the largest eigenvalue of $C_{v}(x, y)$, being proportional to $D(x, y)$, can be used for phase correction.

This eigenvector approach also realizes the maximum likelihood (ML) estimation of $D(x, y)$ as follows. The joint probability density function of a single observation is

$$
p(V(x, y) ; D(x, y))=\pi^{-N}\left|C_{V}(x, y)\right|^{-1} \exp \left\{-V^{\mathrm{H}}(x, y) C_{V}^{-1}(x, y) V(x, y)\right\}
$$

It is assumed that the individual pixel vectors in the subimage are independent and have the same covariance matrix. Thus the joint probability density function of 
$V=\left[V\left(x_{1}, y_{1}\right), \ldots, V\left(x_{K L}, y_{K L}\right)\right]$ is the product of probability density function for individual pixel vectors and is given by

$$
p(V ; D(x, y))=\pi^{-N K L}\left|C_{V}(x, y)\right|^{-} K L \exp \left\{-K L \operatorname{Tr}\left(C_{V}^{-1}(x, y) \hat{C}_{V}(x, y)\right)\right\}
$$

where

$$
\hat{C}_{V}(x, y)=\frac{1}{K L} \sum_{i=1}^{K L} V\left(x_{i}, y_{i}\right) V^{\mathrm{H}}\left(x_{i}, y_{i}\right)
$$

$\operatorname{Tr}($ ) the trace of matrix, $K$ and $L$ are the dimensions of the SAR subimage in range and azimuth, respectively. Maximizing the log joint probability density function with respect to the unknown parameter $D(x, y)$ results in the following equation in the appendix (Gray et al. 1989)

$$
\hat{C}_{V}(x, y) D_{\mathrm{ML}}(x, y)=\lambda_{\max } D_{\mathrm{ML}}(x, y)
$$

where $\lambda_{\max }$ is the maximal eigenvalue of $\hat{C}_{V}(x, y), D_{\mathrm{ML}}(x, y)$ is the corresponding eigenvector and the ML estimation of $D(x, y)$. Equation (34) shows that the eigenvector corresponding to the maximal eigenvalue of $\hat{C}_{V}(x, y)$ is the ML phase estimation of $D(x, y)$.

\section{Elevational imaging}

Three-dimensional SAR imaging is able to produce a radar image as a function of range, azimuth and elevation. High-range resolution is achieved by pulse compression of the transmitted wideband signals. Azimuthal and elevational resolutions rely on the synthesized apertures in azimuth and elevation, respectively. In this section, phased array beamforming techniques based on frequency domain beamforming are used to obtain the elevational resolution by forming many receiving beams in the elevational direction. Super-resolution processing is used to enhance the elevational resolution.

\subsection{Elevational processing}

After phase correction and resampling, all the flight paths are equi-spaced and an elevational beam is formed in the broadside direction of the flight path array. Thus the signal from this direction is enhanced. However, signals from other directions are not in phase and are not reinforced. To form an image in these directions, the complex image of each flight path is required to be shifted in phase to compensate the difference of propagation path in order to sum them coherently. Due to the fact that the variation of azimuthal aspect angle within a subimage is negligible, the difference of propagation path for $i$ th flight path with respect to the first flight path as shown in figure 4 is

$$
\Delta r_{i l}=(i-1) d \sin \theta_{l}
$$

where $d$ is the baseline between adjacent flight paths and $\theta_{l}$ the elevational signal direction. Thus the corresponding phase shift is expressed as

$$
\eta_{i l}=4 \pi(i-1) d \sin \theta_{l} / \lambda
$$

due to two-way propagation. The frequency-domain beamforming approach steers elevational receiving beam by varying $\eta_{i l}$ as shown in figure 4 . Two groups of phase shifters are illustrated. The first group conducts the phase correction to compensate for the unknown array aperture as discussed in $\S 5$. The second group applies a linear 


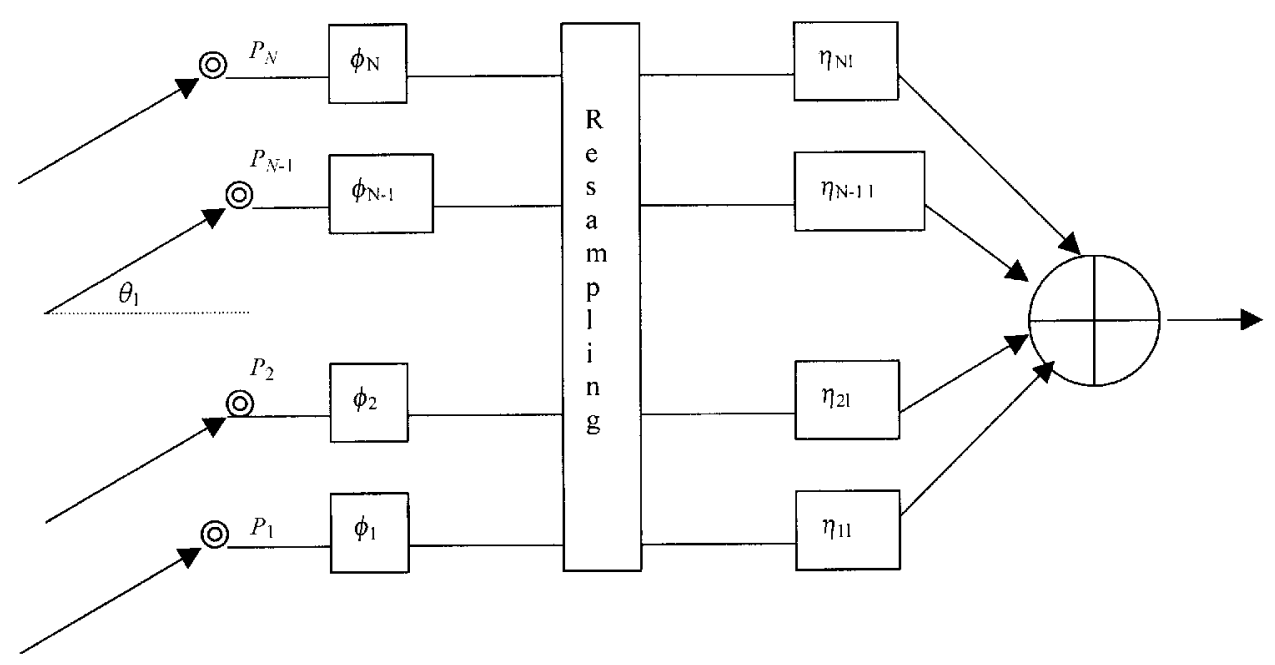

Figure 4. Focusing and scanning of elevational beam.

phase across the equi-spaced flight path array to scan the focused beam electronically in the elevational direction.

For an equi-spaced flight path array, the steering phase factor is $\exp \left\{-j 4 \pi(i-1) d \sin \theta_{l} / \lambda\right\}$. By use of relation $\sin \theta_{l} \approx z / R_{0}$ the output of the elevational beamformer is

$$
\hat{\sigma}(x, y, z)=\sum_{i=1}^{N} G_{i}^{\prime}(x, y) \exp \left\{-j 4 \pi(i-1) d z /\left(\lambda R_{0}\right)\right\}
$$

where $G_{i}^{\prime}(x, y)=G_{i}(x, y) \exp \left(j \phi_{i}\right)$ denotes the complex image after phase correction and resampling. Equation (37) can be computed efficiently using an $N$-point FFT. Use of an $N$-point FFT produces multiple receiving beams at elevational height $z$ given by

$$
z=\lambda l R_{0} /(2 d N)|l|<N / 2
$$

The shading weight coefficients $b_{i} i=1, \ldots, N$ are generally applied to the individual phase corrected and resampled images to control the mainlobe width and sidelobe levels of elevational beams. In order to increase the number of elevational beams steered, the windowed, phase-corrected and resampled images $b_{i} G_{i}^{\prime}(x, y) i=1, \ldots, N$ need to be appended with zeros prior to FFT of the extended data (Kay and Marple 1981).

\subsection{Super-resolution processing}

Super-resolution has been extensively applied to 2D SAR imaging (DeGraaf 1998). In this section it is extended to 3D SAR imaging. Whilst zero-padding increases the number of steered elevational beams, the elevational resolution remains unchanged because zero-padding does not increase the array aperture. The elevational resolution is determined by the aperture length in elevation, which is known as Rayleigh resolution. The Rayleigh resolution can only be enhanced by increasing the effective aperture length, which is impractical for multiple-pass space-borne SAR processing. Super-resolution processing of multiple-pass SAR allows the Rayleigh 
resolution to be overcome and consequently to be preferred as it reduces the number of flight paths for a specified elevational resolution and thus saves the experimental expense and time and mitigates the decorrelation of SAR images.

The model of multiple complex sinusoids in equation (37) allows modern spectral estimation to be used successfully to increase the spectral resolution beyond the Rayleigh resolution. Such techniques (Stoica and Moses 1997) include maximal entropy (ME), auto-regressive (AR), moving average (MA), auto-regressive moving average (ARMA), multiple signal classification (MUSIC) and estimation of signal parameters via rotational invariance techniques (ESPRIT). We applied the MUSIC algorithm to elevational imaging (She et al. 1999). However, it requires that the number of signals be estimated a priori so that the accurate separation of signal and noise subspaces of the covariance matrix can be realized (Schmidt 1986) and the estimation of the covariance matrix with spatial smoothing usually reduces the resolution of 3D image in range and azimuthal directions (Williams et al. 1988).

In this section, a super-resolution approach, which is robust to signal model selection, is used to enhance the elevational resolution. The block diagram of elevational super-resolution is shown in figure 5. After image registration, phase correction and flight path resampling, the data across multiple SAR images with the same pixel position, that is the output of flight path array at this pixel, form a pixel series. Instead of DFT processing, maximal entropy data extrapolation is applied to each pixel series to enhance the elevational resolution.

The philosophy of maximal entropy spectral estimation is that all extrapolations of the measured data should be consistent with the available data and should make minimal assumption regarding unavailable data. For a 1D stationary Gaussian process, maximal entropy is equivalent to the AR process and there are two main ways to estimate the AR spectrum. The first approach is to estimate the prediction coefficients and to calculate the spectrum using the estimated prediction coefficients. The second approach also estimates the prediction coefficients, but then extrapolates the observed data in both directions with the estimated prediction coefficients, and finally applies a Fourier transform to the extrapolated data. Since both approaches

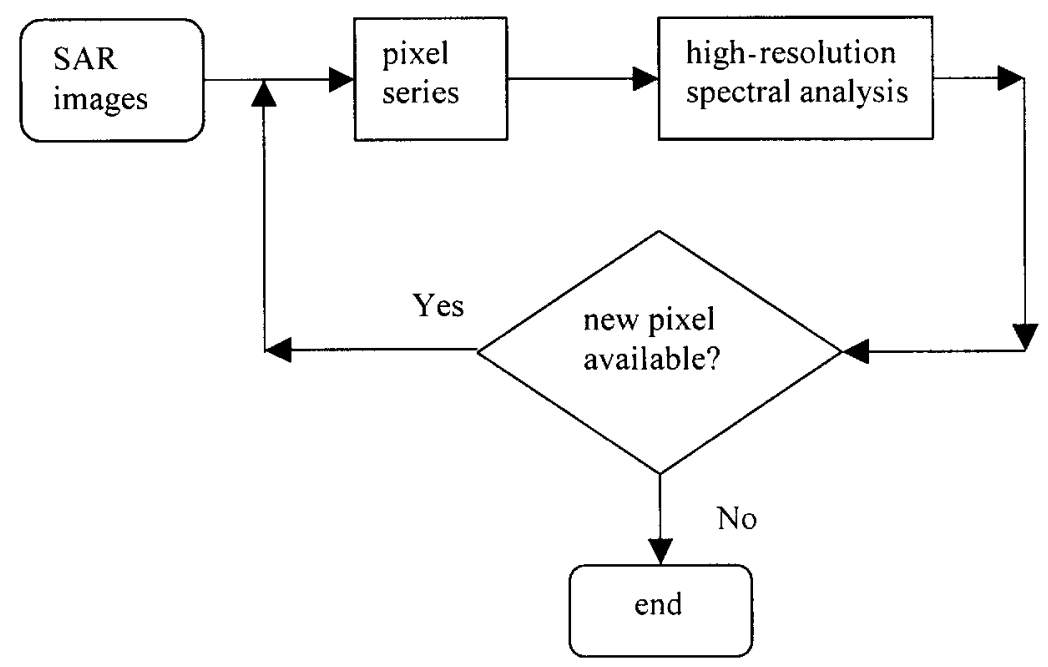

Figure 5. Elevational super-resolution processing. 
use the same prediction coefficients, they should provide essentially the same spectral estimation. However, the first approach only estimates the power spectrum and ignores the phase spectrum. The second approach, despite being more computationintensive, produces both the power and phase spectra and this is sometimes useful for radar and sonar applications (Swingler and Walker 1989, Wu 1995). Moreover this data extrapolation approach is insensitive to the order of model. Thus the second approach is employed.

The proposed super-resolution includes three steps. First the Burg algorithm is applied to the pixel series $G_{i}^{\prime}(x, y)$ at pixel $(x, y)$ to estimate the prediction coefficients $h_{Q}(x, y, k) k=1, \ldots, Q$ where $Q$ is the AR model order. Then the pixel series is extrapolated forward and backward to the length $N F$ where $F$ is a factor of extrapolation. The forward extrapolation of data is given by

$$
G_{N+i}^{\prime}(x, y)=\sum_{k=1}^{Q} h_{Q}(x, y, k) G_{N^{+i^{-}} k}^{\prime}(x, y) i>0
$$

and the backward extrapolation of data is done by

$$
G_{-i}^{\prime}(x, y)=\sum_{k=1}^{Q} h_{Q}^{*}(x, y, k) G_{k^{-} i}^{\prime}(x, y) i>0
$$

Finally the extrapolated pixel series is Fourier transformed to produce the superresolution.

The selection of model order is a critical step. If the model is too low, the spectrum has all the peaks smoothed. On the other hand, if the order is too large, the spectrum may contain spurious peaks. Many criteria such as Akaike Information Criterion (AIC) and Minimum Description Length (MDL) have been proposed to estimate the model order (Stoica and Moses 1997). An effective approach to determine the model order $Q$ of AR for the short data in radar application is (Wu 1995)

$$
Q=N / 3 \sim N / 2
$$

and the extrapolation factor is selected as

$$
F=2 \sim 4
$$

\section{Experimental results}

\subsection{Simulated data}

The computer simulations were conducted based on the system parameters of ERS-1 where the satellite operated at an altitude of $785 \mathrm{~km}$. The radar transmitted a linear-frequency-modulated (LFM) signal with a bandwidth of $15.55 \mathrm{MHz}$ and a nominal wavelength of $5.67 \mathrm{~cm}$. A point scatterer was located in a flat terrain. Seventeen flight passes spaced $100 \mathrm{~m}$ apart vertically as shown in figure 6 were simulated. The distribution of flight passes provided an elevational aperture of $1600 \mathrm{~m}$, which is similar to that of ERS-1 real data.

At first a matched filter was applied to obtain the SAR complex image of each flight path. Then 17 SAR images were registered with image model matching, corrected in phase by the eigenvector method, and resolved in the elevational direction with a 32-point FFT. Figure 7 shows the 3D image of the scatterer illustrating the $2 \mathrm{D}$ point-spread-functions (PSF) with $(a)$ in the range-elevation plane, $(b)$ in the azimuth-elevation plane, and $(c)$ in the range-azimuth plane. The $3 \mathrm{D}$ image is $32 \times 32 \times 32$ pixels. Each pixel has a size of $9.38 \mathrm{~m}, 13.79 \mathrm{~m}$ and $6.90 \mathrm{~m}$ in the range, azimuthal and elevational directions. The theoretical resolutions in the range, 

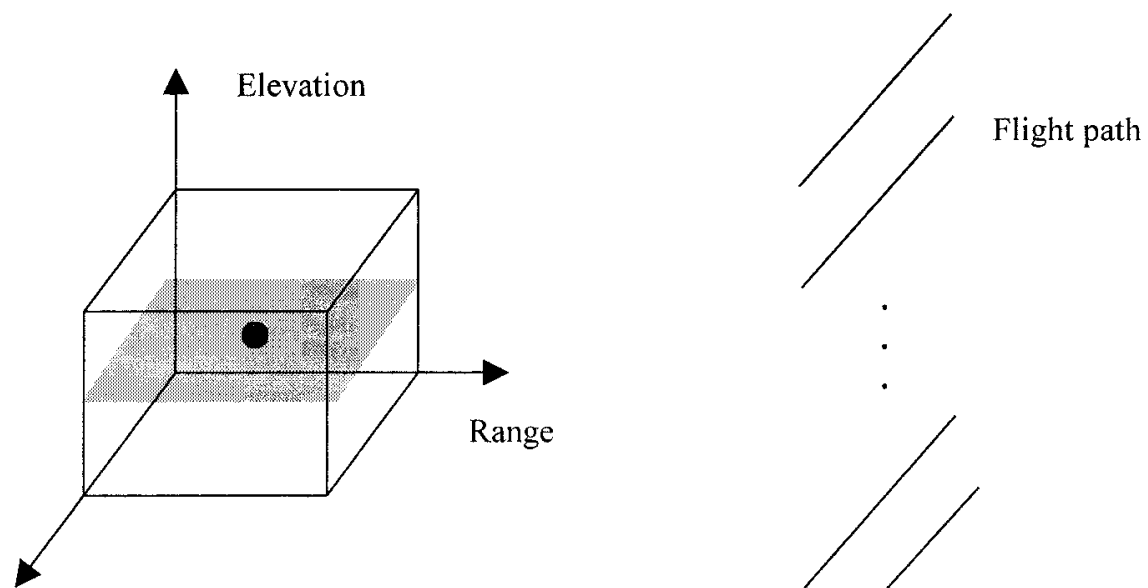

Azimuth

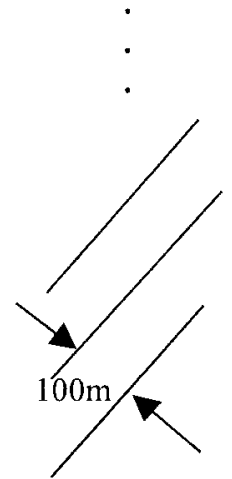

Figure 6. Simulation of 3D SAR imaging.

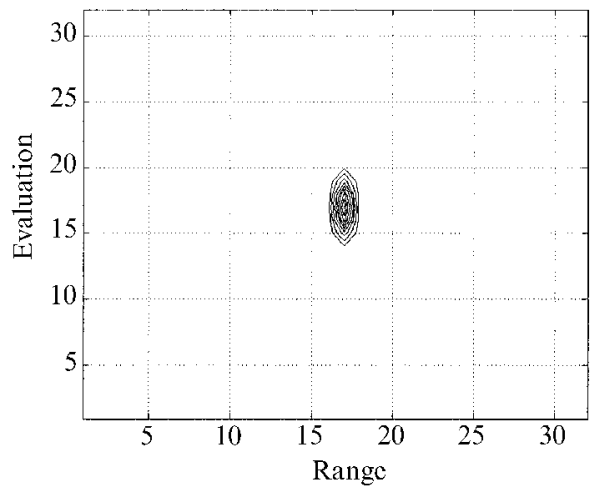

(a)

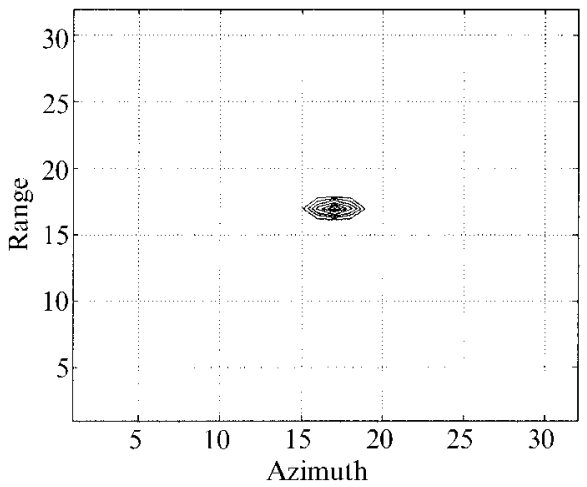

(c)

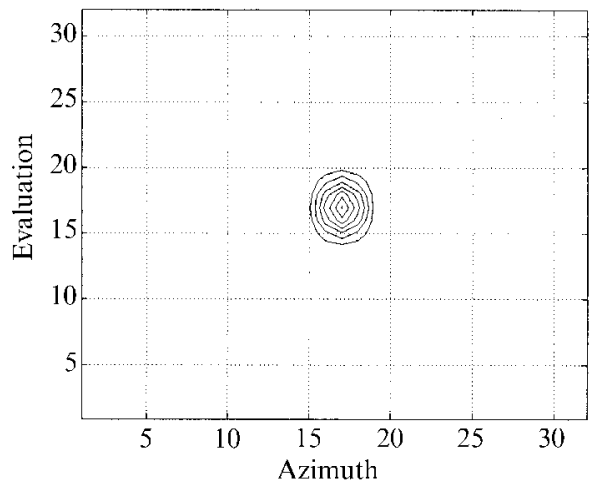

(b)

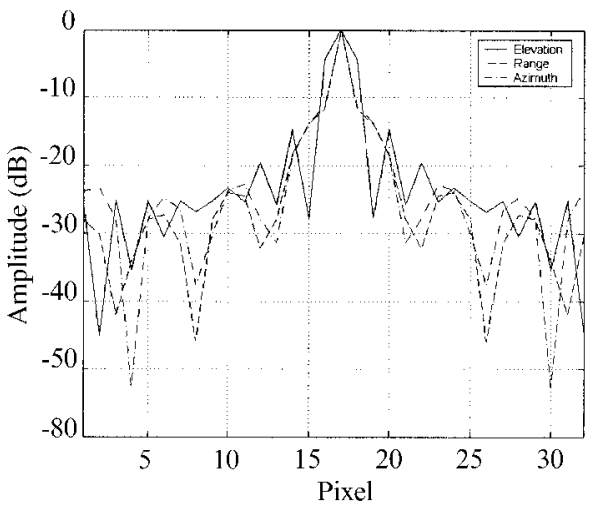

(d)

Figure 7. 3D image of simulated data. 
azimuth and elevation are $9.38 \mathrm{~m}, 13.79 \mathrm{~m}$ and $13.79 \mathrm{~m}$, respectively. The maximal unambiguous distance in the elevation is $222.55 \mathrm{~m}$.

Resolution and noise are two important performance measures for an imaging system since both impact the capability to perform image reconstruction. They are characterized by the PSF of the imaging system. Resolution is characterized by the $3 \mathrm{~dB}$ width of the main lobe of the PSF while noise is characterized by the height of the sidelobes. The 1D PSFs in range, azimuth and elevation are given in figure $7(d)$ which show that the MPSAR processing is able to provide the focused resolutions in range, azimuth and elevation, the measured resolutions ( $3 \mathrm{~dB}$ width of PSF) in range, azimuthal and elevational directions are consistent with the theoretical resolutions and the peak sidelobes have a level of $-24 \mathrm{~dB},-23 \mathrm{~dB}$ and $-15 \mathrm{~dB}$ in range, azimuth and elevation directions.

In order to confirm the validity of the proposed approaches to natural scattering, a volume area having a size of $128 \mathrm{~m} \times 128 \mathrm{~m}$ and a random normal distribution of reflectivity in the range-elevation plane was simulated and is shown in figure $8(a)$. The other parameters were the same as those of a point scatterer described above. The imaging result after image registration, phase correction, resampling and elevational processing is given in figure $8(b)$. It is seen that although some distortions exist between figure $8(a)$ and $(b)$ (Mitchell 1974), figure $8(b)$ has a correspondence to the scatterer distribution in figure $8(a)$ and the scatterers in figure $8(b)$ are not well resolved in the elevational direction as the elevational resolution is limited. Then the super-resolution processing was applied to the same data in figure $8(b)$. The data in each range cell were extrapolated forward and backward from 17 to 32 . The order of $\mathrm{AR}$ model was chosen as $N / 3 \cong 5$. The super-resolution image is shown in figure $8(c)$. The enhancement of elevational resolution is obvious.

\subsection{ERS-1 real data}

The multiple image registration, the phase correction and the elevational imaging were tested using nine SAR image datasets, acquired by the ERS-1 satellite over a small area in the south-west of the city of Bonn, Germany, during the period 2-29 March 1992. The nine flight paths were parallel and their elevational positions are shown in figure 9 where the four digit numbers located in the right above each flight path point are the ERS-1 orbit numbers. The nine flight paths provided a synthesized aperture length of $1686 \mathrm{~m}$. An array of 19 corner reflectors was deployed by the Institute of Navigation, University of Stuttgart, in the terrain for calibration.

An experimental study was carried out to measure the performances of the image

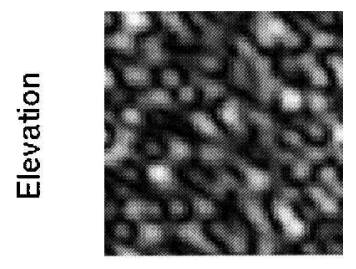

Range

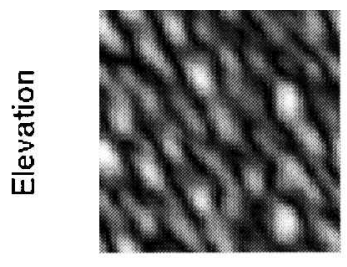

Range

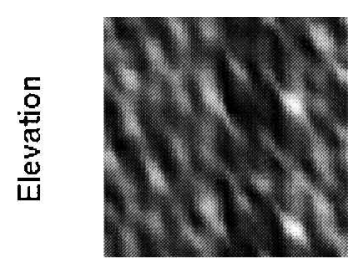

Range

(a)

(b)

(c)

Figure 8. Simulation of random scattering. 


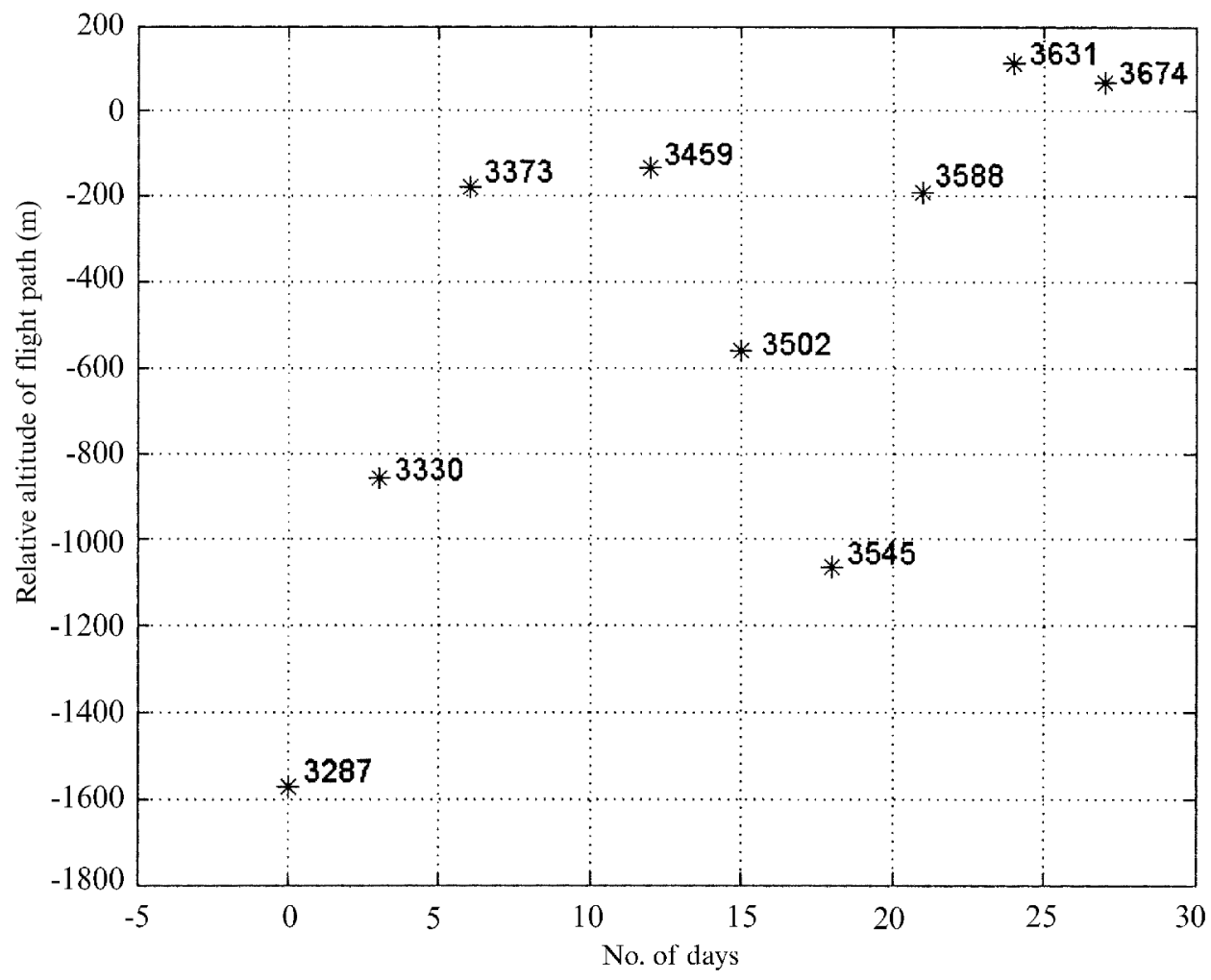

Figure 9. Relative elevational positions of ERS-1 flight paths.

model matching approach using multiple SAR subimages of the same terrain. The similarity of multiple SAR images is determined by the signal-to-noise ratio (SNR) of SAR image, the baseline of flight path and the time interval of flight path (Zebker and Villasenor 1992). It increases with an increase of SNR or a decrease of baseline and time interval. The amplitude correlation method, being a standard method, was used as a comparison. The experiment included 162 pairs of SAR image datasets by choosing subregions with a size of $50 \times 50$ pixels. The translational offset of two images within each pair was known to within a pixel by the use of ground control points. For each offset, a window size of $32 \times 32$ was selected in the calculation of the registration measure. This allowed for offsets of up to \pm 9 pixel positions in each direction. The distance measure between image pairs $G_{i}(x, y)$ and $G_{l}(x, y)$ was chosen as

$$
\mathrm{D}\left[G_{i}(x, y), G_{l}(x, y)\right]=\sum_{x} \sum_{y}\left[\left|G_{i}(x, y)\right|-\left|G_{l}(x, y)\right|\right]^{2}
$$

The reference image was updated by the incoherent summation, that is,

$$
\operatorname{sum}[M(x, y), G(x, y)]=|M(x, y)|+\gamma|G(x, y)|
$$

where $\gamma$ is the normalized correlation coefficient between $|M(x, y)|$ and $|G(x, y)|$. Figure 10 gives the correct probability of images registration using the image model matching approach (solid line) and the amplitude correlation (dashed line). It shows 


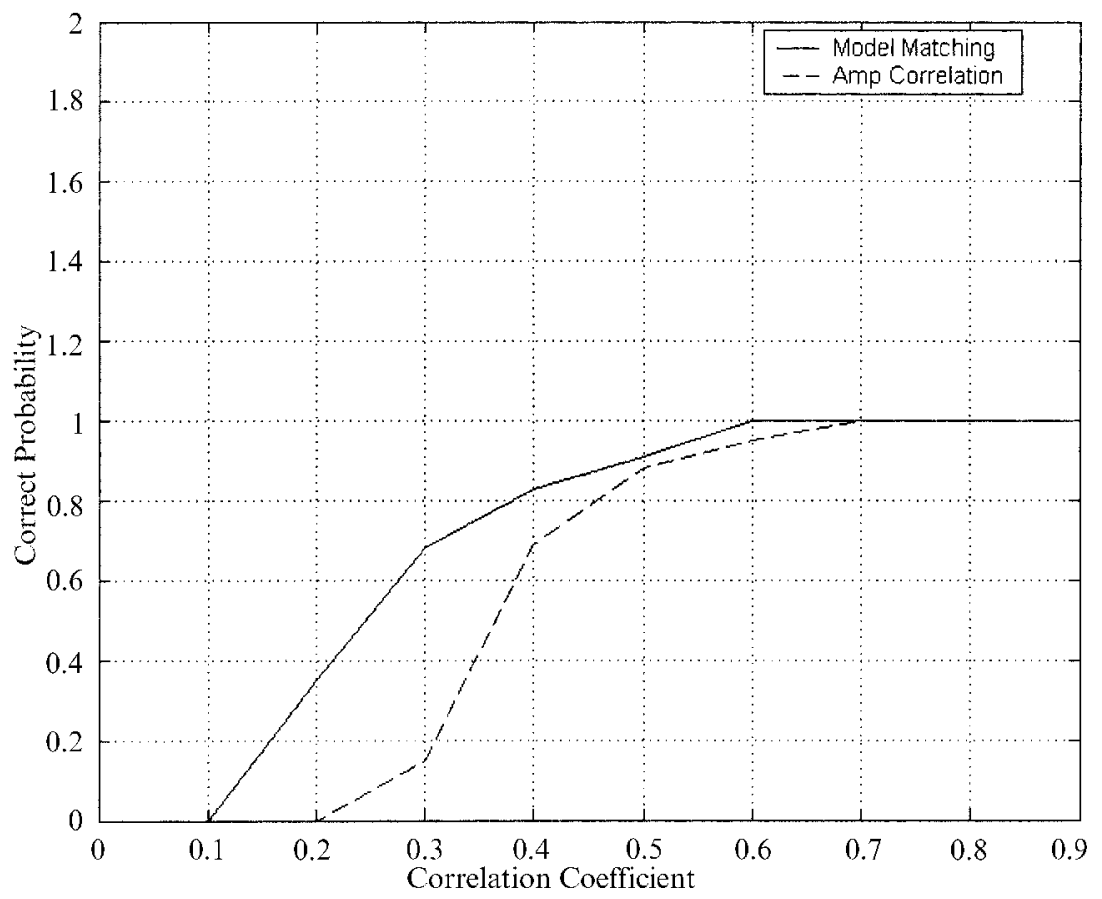

Figure 10. Registration accuracy versus image similarity.

that both provide correct registration if image similarity is greater than 0.6 for the image model matching approach and 0.7 for the amplitude correlation. However, the image model matching degrades more slowly as the correlation coefficient decreases. The thresholds of registration accuracy $80 \%$ for the image model matching and the amplitude correlation are 0.37 and 0.46 , respectively.

In figure 11, the dashed line denotes the correlation coefficient histogram of image pairs and the solid line is the correlation coefficient histogram between the reference image and each image. It is seen that the reference image has a better correlation with multiple images and so the image model approach can increase the accuracy of multiple image registration.

An image patch containing a corner reflector with $32 \times 32$ pixels was used for 3D SAR processing. Three-dimensional image was formed by registering nine SAR images with the image model matching, correcting them in phase by the eigenvector method to remove the derivation of flight path, and elevational imaging where the unequal spaced flight paths were interpolated into equi-spaced flight paths and then the FFT was conducted. Figure 12 gives the 2D slices of 3D image of terrain. The $3 \mathrm{D}$ image is $32 \times 32 \times 16$ pixels. Each pixel has a size of $7.90 \mathrm{~m}, 4.00 \mathrm{~m}$ and $6.73 \mathrm{~m}$ in the range, azimuthal and elevational directions. The resolutions in the range, azimuth and elevation are $9.38 \mathrm{~m}, 6.00 \mathrm{~m}$ and $13.45 \mathrm{~m}$, respectively. The focused resolution of the MPSAR processing in the elevation is illustrated in figure 12. In order to display the weak scattering, the display range of $3 \mathrm{D}$ image is selected from $-30 \mathrm{~dB}$ to $0 \mathrm{~dB}$.

The effects of image registration and phase correction were investigated. Figure 13 gives the 3D image formed with elevational imaging and without image registration and phase correction. It indicates that profiles in range-elevation and azimuthelevation planes are out of focus in the elevational direction and signals resulting 


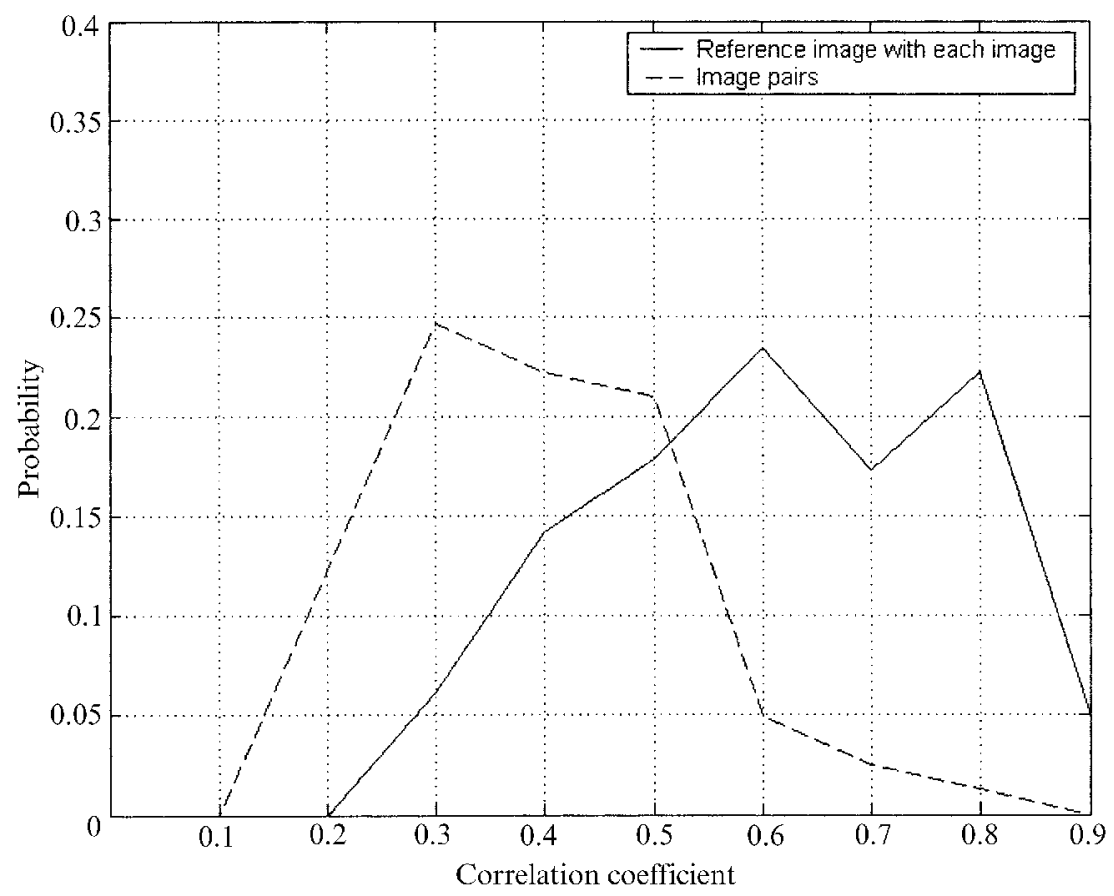

Figure 11. Histogram of correlation coefficients.
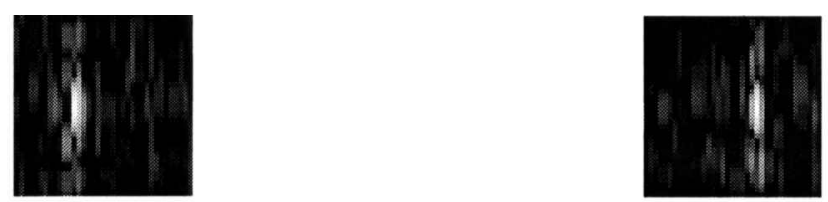

(a) Range-elevation plane

(b) Azimuth-elevation plane

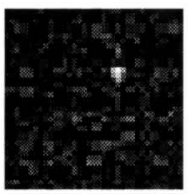

(c) Azimuth-range plane

Figure 12. 3D image of ERS-1 data.

from the corner reflector are distributed in several range-azimuth cells in rangeazimuth plane. The 3D image formed with image registration and elevational imaging and without phase correction is given in figure 14. It is seen that signals corresponding to the corner reflector are located in the same range-azimuth cells. However, they are still unfocused and scattered over the elevational direction. Figures 12, 13 and 


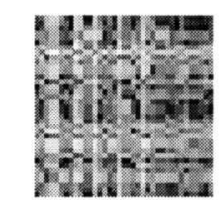

(a) Range-elevation plane

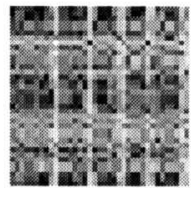

(b) Azimuth-elevation plane

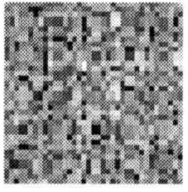

(c) Azimuth-range plane

Figure 13. 3D image of ERS-1 data without image registration and phase correction.

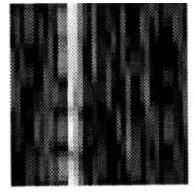

(a) Range-elevation plane

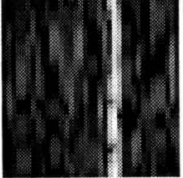

(b) Azimuth-elevation plane

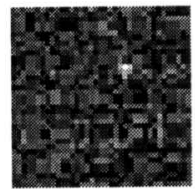

(c) Azimuth-range plane

Figure 14. 3D image of ERS-1 data without phase correction.

14 illustrate that image registration, phase correction and elevational imaging are the essential processing steps for 3D SAR imaging.

Instead of elevational imaging with FFT, the 3D image formed by the superresolution method for elevational resolution enhancement is shown in figure 15. The pixel vectors were extrapolated forward and backward from 9 to 32. The AR model order was selected as $N / 3=3$. The enhancement of elevational resolution is obvious.

A large patch of terrain with a size of $256 \times 256$ pixels was chosen for $3 \mathrm{D}$ SAR imaging. The 2D SAR image in the range-azimuth plane is shown in figure 16. It involves the natural scattering from trees and buildings. This large image was divided 


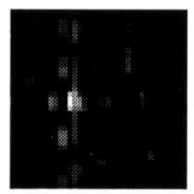

(a) Range-elevation plane

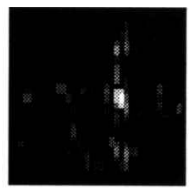

(b) Azimuth-elevation plane

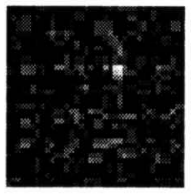

(c) Azimuth-range plane

Figure 15. 3D image of ERS-1 data with elevational super-resolution.

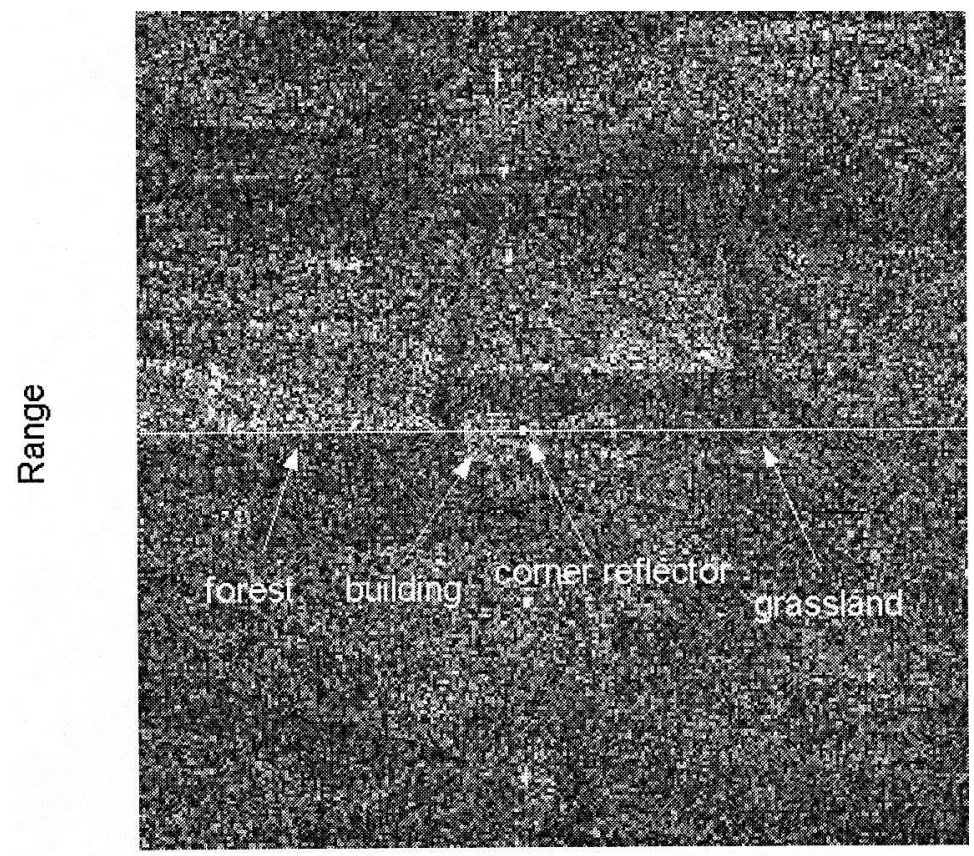

\section{Azimuth}

Figure 16. 2D SAR image of large area with a marked profile.

into subimages with a size of $32 \times 32$ pixels. After the $3 \mathrm{D}$ image for each subimage was computed, the 3D image of the large area was reconstructed by mosaicking the $3 \mathrm{D}$ images of the subimages. Profiles of the large $3 \mathrm{D}$ image in the azimuth-elevation 
plane are shown in figure 17 where $(a)$ is the Fourier processing and $(b)$ is the superresolution imaging. As the position of the profile is marked in figure 16 a corner reflector on the top of the building is in the middle, two small trees on a grassland are located in the right and a forest of tall trees is in the left. Locations of the building, the corner reflector, the forest and the small trees are plotted and superimposed over the SAR image in figure 17(b). By comparing figure 17(b) with $(a)$ the improvement of elevational resolution is impressive. But a small slope of forest and building roof exists in figure $17(a)$ and $(b)$. It is the inherent geometrical distortion in space-borne SAR imagery (Curlander 1984), which is caused by the SAR imaging geometry and the Earth rotation. Despite this inherent defect, figure $17(a)$ and $(b)$ have a correspondence to the scene. By use of equation (38) the height difference between the forest and the small trees is about $11 \mathrm{~m}$, which has an agreement with manual measurement.

In order to measure the 3D image quality of ERS-1 data quantitatively, three elevational profiles of the corner reflector in figure 17 without calibration, with calibration and with super-resolution are presented in figure 18. They are characterized by $3 \mathrm{~dB}$ bandwidth (BW), peak sidelobe level (PSLR) and integrated sidelobe level (ISLR). BW determines the measured elevational resolution. PSLR is the ratio of the highest sidelobe value to the mainlobe value. The smaller the PSLR, the smaller the sidelobe level. ISLR is defined as the ratio of the integrated sidelobe energy to the mainlobe energy. The smaller the ISLR, the smaller the sidelobes and the better the resolution capability. BW, PSLR and ISLR of these three profiles were

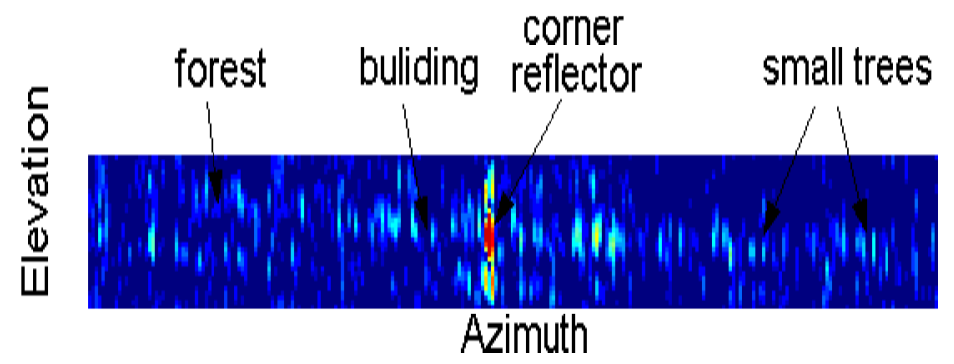

(a) Fourier processing

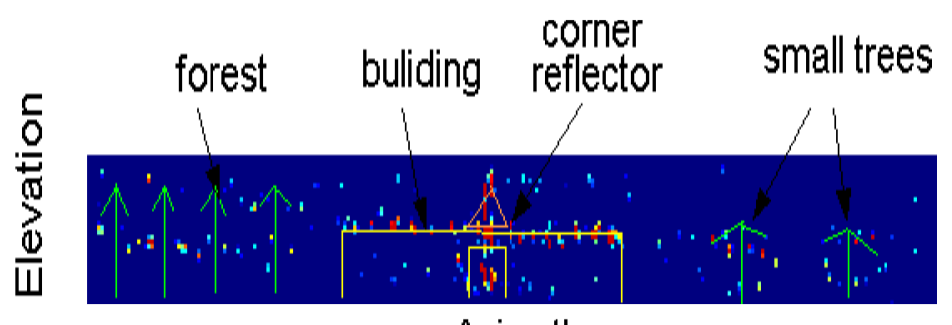

Azimuth

(b) Super-resolution processing

Figure 17. Profiles of 3D SAR image in the azimuth-elevation plane. 


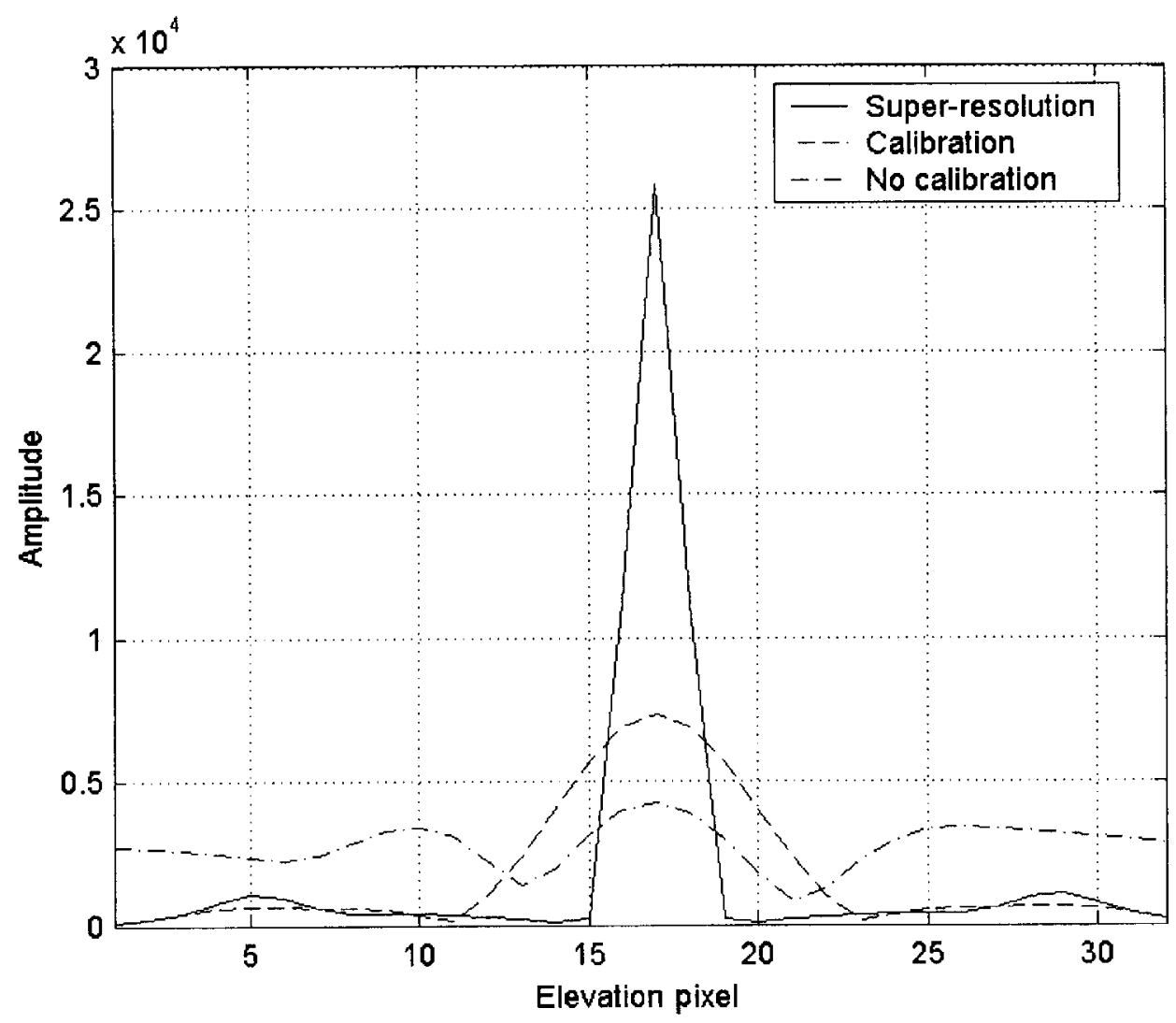

Figure 18. Elevational profiles of corner reflector.

calculated and are listed in table 1. It indicates that calibration obtains a gain of $19 \mathrm{~dB}$ for PSLR and $14 \mathrm{~dB}$ for ISLR and super-resolution provides an improved factor of larger than three for elevational resolution and a gain of $6 \mathrm{~dB}$ for PSLR and $9 \mathrm{~dB}$ for ISLR.

\section{Conclusions}

In this paper, a novel approach for 3D space-borne SAR imaging via multiplepass processing is developed based on the mathematical model of 3D SAR imaging. It calibrates the uncertainty of flight path with the image registration and the phase correction and images in the elevation with multiple focused beams. The image model matching is proposed for multiple image registration, the eigenvector method is applied for the phase correction and the super-resolution method is utilized for enhancement of elevational resolution. Super-resolution is preferable to reduce the

Table 1. Parameters of elevational profiles of corner reflector.

\begin{tabular}{lccr}
\hline Elevational profiles & BW $(\mathrm{m})$ & PSLR $(\mathrm{dB})$ & ISLR $(\mathrm{dB})$ \\
\hline No calibration & 20 & -2 & 11.64 \\
Calibration & 20 & -21 & -2.52 \\
Super-resolution & 6 & -27 & -11.55 \\
\hline
\end{tabular}


number of flight paths of the SAR system and thus mitigate the decorrelation of SAR images. Three-dimensional images of the simulated and real ERS-1 data are produced with the proposed approaches. The processing results of ERS-1 data indicate that the improved factor of elevational super-resolution is larger than three, PSLR and ISLR are reduced significantly by the calibration of flight path and they are further enhanced by use of the super-resolution processing. The generated 3D images demonstrate the promise of developed approaches for applications in topography surveying, forest and crops monitoring and concealed target detection.

\section{Acknowledgments}

The authors would like to thank the anonymous referees and editor Dr S. Tanaka for their constructive comments to improve the quality of this paper. We would also like to thank Dr Thiel from Insitut für Navigation, University of Stuttgart, as well as the European Space Agency for providing the ERS-1 data used in the experimental study. The first author would like to acknowledge the scholarships from the Australian Research Council, the University of Adelaide and CSSIP for his PhD study at the University of Adelaide.

\section{Appendix: Derivation of (34)}

The joint probability density function is

$$
p(V ; D(x, y))=\pi^{-N K L}\left|C_{V}(x, y)\right|^{-} K L \exp \left\{-K L \operatorname{Tr}\left(C_{V}^{-1}(x, y) \hat{C}_{V}(x, y)\right)\right\}
$$

where

$$
\hat{C}_{V}(x, y)=\frac{1}{K L} \sum_{i=1}^{K L} V\left(x_{i}, y_{i}\right) V^{\mathrm{H}}\left(x_{i}, y_{i}\right)
$$

is an estimator of covariance matrix $C_{V}(x, y)$. Maximizing the logarithm joint probability density function with respect to $D(x, y)$ leads to minimization of

$$
l=K L \ln \left|C_{v}(x, y)\right|+K L \operatorname{Tr}\left(C_{v}^{-1}(x, y) \hat{C}_{v}(x, y)\right)
$$

By use of relations (Stoica and Moses 1997)

$$
\frac{\partial\left|C_{v}(x, y)\right|}{\partial D(x, y)}=\left|C_{v}(x, y)\right| \operatorname{Tr}\left(\frac{\partial C_{v}(x, y)}{\partial D(x, y)} C_{v}^{-1}(x, y)\right)
$$

and

$$
\frac{\partial C_{v}^{-1}(x, y)}{\partial D(x, y)}=-C_{v}^{-1}(x, y) \frac{\partial C_{v}(x, y)}{\partial D(x, y)} C_{v}^{-1}(x, y)
$$

we have

$$
\frac{\partial l}{\partial D(x, y)}=K L \operatorname{Tr}\left\{\frac{\partial C_{v}(x, y)}{\partial D(x, y)}\left[C_{v}^{-1}(x, y)-C_{v}^{-1}(x, y) \hat{C}_{v}(x, y) C_{v}^{-1}(x, y)\right]\right\}
$$

Based on equation (29), we get

$$
\frac{\partial C_{v}(x, y)}{\partial D^{\mathrm{H}}(x, y)}=\left|G_{1}(x, y)\right|^{2} D(x, y)
$$

that is,

$$
\frac{\partial C_{v}(x, y)}{\partial(D(x, y))_{n}}=\left|G_{1}(x, y)\right|^{2} D(x, y) \delta_{n}^{T}
$$


where $\delta_{n}=(0,0, \ldots, 0,1,0, \ldots, 0)^{T} n=1, \ldots, N$. Setting

$$
\frac{\partial l}{\partial(D(x, y))_{n}}=0
$$

the maximum likelihood estimation $D_{\mathrm{ML}}(x, y)$ satisfies

$$
\operatorname{Tr}\left[\left|G_{1}(x, y)\right|^{2} D_{\mathrm{ML}}(x, y) \delta_{n}^{T}\left(C_{v}^{-1}(x, y)-C_{v}^{-1}(x, y) \hat{C}_{v}(x, y) C_{v}^{-1}(x, y)\right)\right]=0 \quad n=1, \ldots, N
$$

Equation (A8) can be changed into

$$
\delta_{n}^{T} C_{v}^{-1}(x, y) D_{\mathrm{ML}}(x, y)-\delta_{n}^{T} C_{v}^{-1}(x, y) \hat{C}_{v}(x, y) C_{v}^{-1}(x, y) D_{\mathrm{ML}}(x, y)=0 \quad n=1, \ldots, N
$$

which is equivalent to

$$
\hat{C}_{v}(x, y) C_{v}^{-1}(x, y) D_{\mathrm{ML}}(x, y)=D_{\mathrm{ML}}(x, y)
$$

By use of the matrix inverse

$$
C_{v}^{-1}(x, y)=\sigma_{w}^{-}{ }^{2}\left[I-\frac{\left|G_{1}(x, y)\right|^{2} D(x, y) D^{H}(x, y)}{\sigma_{w}^{2}+\left|G_{1}(x, y)\right|^{2} N}\right]
$$

we have

$$
\hat{C}_{v}(x, y) D_{\mathrm{ML}}(x, y)=\lambda_{\max } D_{\mathrm{ML}}(x, y)
$$

where $\lambda_{\max }$ is the maximal eigenvalue of $\hat{C}_{v}(x, y)$.

\section{References}

Ausherman, D. A., Kozma, A., Walker, J. L., Jones, H. M., and Poggio, E. C., 1984, Developments in radar imaging. IEEE Transactions on Aerospace and Electronic Systems, 20, 363-398.

Chan, C. K., and Farhat, N. H., 1981, Frequency swept tomographic imaging of threedimensional perfectly conducting objects. IEEE Transactions on Antenna Propagation, 29, 312-319.

Curlander, J. C., 1984, Utilization of spaceborne SAR data for mapping. IEEE Transactions on Geoscience and Remote Sensing, 24, 106-112.

DeGraAf, S. R., 1998, SAR imaging via modern 2D spectral estimation methods. IEEE Transactions on Image Processing, 7, 729-761.

FARHat, N. H., 1984, Prospects for three-dimensional projective and tomographic imaging radar networks. Radio Science, 19, 1347-1355.

Fortuny, J., and Sieber, A. J., 1999, Three-dimensional synthetic aperture radar imaging of a fir tree: first results. IEEE Transactions on Geoscience and Remote Sensing, 37, 1006-1014.

Gabriel, A. K., and Goldstein, R., 1988, Crossed orbit interferometry: theory and experimental results from SIR-B. International Journal of Remote Sensing, 9, 857-872.

Gatelli, F., Guarnieri, A. M., Parizzi, F., Pasquali, P., Prati, C., and Rocca, F., 1994, The wavenumber shift in SAR interferometry. IEEE Transactions on Geoscience and Remote Sensing, 32, 855-865.

Goldstein, R. M., ZebKer, H. A., and Werner, C. L., 1988, Satellite radar interferometry: two-dimensional phase unwrapping. Radio Science, 23, 713-720.

Graham, L. C., 1974, Synthetic interferometer radar for topographic mapping. Proceedings of IEEE, 62, 763-768.

Gray, D. A., Wolfe, W. O., and Riley, J. L., 1989, An eigenvector method for estimating the positions of the elements of an array of receivers. Proceedings of the Australian Symposium on Signal Processing Application, Adelaide, Australia (Adelaide: IEEE), pp. 391-393. 
Homer, J., Longstaff, I. D., and Callaghan, G., 1996, High resolution 3D SAR via multibaseline interferometry. Proceedings of IGARSS, Lincoln, NE, 27-31 May 1996 (Noordwijk: ESA), pp. 796-798.

Homer, J., LongstafF, I. D., and Zhishun She, 1997, Improved digital elevation models via multi-baseline interferometric SAR. Proceedings of IGARSS, Singapore, 3-8 August 1997 (Noordwijk: ESA) pp. 1579-1581.

Jakowatz, C. V., Wahl, D. E., Eichel, P. H., Ghiglia, D. C., and Thompson, P. A., 1996, Spotlight-mode Synthetic Aperture Radar: A Signal Processing Approach (Boston: Kluwer).

KaY, S. M., and MARPLE, S. L., 1981, Spectrum analysis-a modern perspective. ProceedingS of IEEE, 69, 1380-1419.

Krim, H., and ViberG, M., 1996, Two decades of array signal processing research: the parametric approach. IEEE Signal Processing Magazine, 13, 67-94.

LI, F. K., and Goldstein, R. M., 1990, Studies of multibaseline spaceborne interferometric synthetic aperture radars. IEEE Transactions on Geoscience and Remote Sensing, 28, 88-97.

LI, J., BI, Z., LIU, Z., and KNAELl, K., 1997, Use of curvilinear SAR for three-dimensional target feature extraction. IEE Proceedings-Radar, Sonar and Navigation, 144, 275-283.

MARECHAL, N., 1995, Tomographic formulation of interferometric SAR for terrain elevation mapping. IEEE Transactions on Geoscience and Remote Sensing, 33, 726-739.

Mitchell, R. L., 1974, Models of extended targets and their coherent radar images. Proceedings of IEEE, 62, 754-758.

Pasquali, P., Prati, C., Rocca, F., Seymour, M., Fortuny, J., Phimer, E., and Sieber, A. J., 1995, A 3D SAR experiment with EMSL data. Proceedings of IGARSS, Florence, 10-14 July 1995 (Noordwijk: ESA), pp. 784-786.

Reigber, A., Moreira, A., and Papathanassiou, K. P., 2000, First demonstration of airborne SAR tomography using multibaseline L-band data. IEEE Transactions on Geoscience and Remote Sensing, 38, 2142-2152.

Rockah, Y., and Schuitheiss, P. M., 1987, Array shape calibration using sources in unknown locations - part I: far-field sources. IEEE Transactions on Acoustics, Speech and Signal Processing, 35, 724-735.

Rufino, G., Moccia, A., and Esposito, S., 1998, DEM generation by means of ERS tandem data. IEEE Transactions on Geoscience and Remote Sensing, 36, 1905-1912.

Schmidt, R. O., 1986, Multiple emitter location and signal parameter estimation. IEEE Transactions on Antenna and Propagation, 34, 276-280.

She, Z., Gray, D. A., Bogner, R. E., and Homer, J., 1999, Three-dimensional synthetic aperture radar (SAR) imaging via multiple pass processing. Proceedings of IGARSS, Hamburg, 28 June-2 July 1999 (Noordwijk: ESA), pp. 2389-2391.

SkolinK, M. I., 1990, Radar Handbook (New York: McGraw-Hill).

Stoica, P., and Moses, R. L., 1997, Introduction to Spectral Analysis (Upper Saddle River, NJ: Prentice-Hall).

Swingler, D. N., and WALKER, R. S., 1989, Line-array beamforming using linear prediction for aperture interpolation and extrapolation. IEEE Transactions on Acoustics, Speech and Signal Processing, 37, 16-30.

WAlker, J. L., 1980, Range-Doppler imaging of rotating objects. IEEE Transactions on Aerospace and Electronic Systems, 16, 23-52.

Wehner, D. R., 1995, High Resolution Radar (Norwood, MA: Artech House).

Williams, R. T., Prasad, S., Mahalanabis, A. K., and Sibnl, L. H., 1988, An improved spatial smoothing technique for bearing estimation in a multipath environment. IEEE Transactions on Acoustics, Speech, and Signal Processing, 36, 425-432.

Wolf, E., 1969, Three-dimensional structure determination of semi-transparent objects from holographic data. Optics Communications, 1, 153-156.

WU, P., 1995, A criterion for radar resolution enhancement with Burg algorithm. IEEE Transactions on Aerospace and Electronic Systems, 31, 897-915.

Zebker, H. A., and Goldstein, R. M., 1986, Topographic mapping from interferometry synthetic aperture radar observation. Journal of Geophysical Research, 91, 4993-4999.

ZebKer, H. A., and Villasenor, J., 1992, Decorrelation in interferometric radar echoes. IEEE Transactions on Geoscience and Remote Sensing, 30, 950-959.

Zebker, H. A., Rosen, P. A., and Hensley, S., 1997, Atmospheric effects in interferometric synthetic aperture radar surface deformation and topographic maps. Journal of Geophysical Research, 102, 7547-7563. 